\title{
Calculation and analysis of thermal stress for high pressure rotor of steam turbine
}

\author{
Jun Jin ${ }^{1, \text { a }}$, Qin Zhou ${ }^{2, b}$, Yonghui Xie ${ }^{2, c}$ \\ ${ }^{1}$ Shanghai marine equipment research institute, No.10, Hengshan Road, Shanghai, 200031, \\ P.R. China \\ ${ }^{2}$ School of Energy and Power Engineering, Xi'an Jiaotong University, No.28, Xianning West \\ Road,Xi'an, Shaan Xi, 710049, P.R. China \\ ajj7282@sina.com, bzhouqin_s@163.com, cyhxie@mail.xjtu.edu.cn
}

Keywords: Steam turbine, High pressure rotor, Thermal stress

\begin{abstract}
According to the flow operating parameters of turbine during the downtime process , the convective heat transfer coefficient values between rotor surface and steam are calculated. The FEM transient temperature analysis model is setup to obtain the temperature distribution of rotor during the downtime process. Considering the centrifugal force and temperature load of rotor during the downtime process, the high temperature strength finite element model is setup using the dual linear intensive model. Then, the stress distribution of turbine rotor during the downtime process is obtained.
\end{abstract}

\section{Introduction}

With the development of economy, the ammount of electricity demand and structure are continuously changing. The task of peak adjustion of electrical system has been more and more severe. At present, fossile power plant undertakes the main peak adjustion task. During the process of peak adjustion, the load of turbine changes dramatically and large temperature gradient is existed inside the rotor which intenses the lifetime wastage. However, the lifetime of turbine rotor determines the lifetime of the whole power plant [1]. As a result, the accurate estimation of turbine lifetime wastage of turbine rotor under real start-stop curve is essential. It is quite important to set up the optimization stretegy to make the power plant more safe and economical [2-4].

\section{Finite element model}

The investigated objective is the high pressure rotor of a steam turbine whose material is $28 \mathrm{CrMoNiV}$. Because the object is an integral-disk rotor, simplification of rotor rim is conducted and the force of blade to the rotor is replaced by equivalent load [5]. Considering the axial symmetry of computational model, two-dimensional model is adopted to simulate the problem to decrease the calculation amount.

Two dimensional Plane 77 elements are adopted to fulfill the mesh as indicated in Fig. 1. The total node number is 21876 and the element number is 21118 . Because large stress concentration is existed in the rotor round corner, rim round corner, and the temperature change of control stage is intense, the thermal stress of these spots will be intense which even exceeds the yield limit resulting in large low cycle fatigue wastage. In this case, the meshes near these areas are made denser to improve the calculation accuracy.



Fig. 1 Overall mesh indication of rotor 


\section{Boundary condition for temperature field calculation}

The initial temperature field during rotor downtime process is the steady-state temperature field. The thermal boundary condition is setup as below:

1. The heat tansfer between outer surface of rotor and steam is assumed as the third bounday condition. The steam temperature is calculated according to sliding operation regulation. The heat transfer coeffient is calculated according the empirical formula by former Soviet Union [6].

2. The side surface of rotor is the truncation surface of rotor where the heat transfer coefficient is very small. So adibatic condition is assumed in these surfaces.

3. Due to the axial symmetry of rotor, the boundary condition of center line of rotor is assumed as adiabatic.

4. The bearing of rotor is assumed as constant temperature which is the first boundary condition type.

\section{Boundary condition for stress field calculation}

The boundary condition of stress field calculation is set up as below:

1. The mechanical property of material is set up. Elastic-Plastic calculation adopt the dual linear intensive model

2. Displacement constraint is set up. Axial displacement constrains are set up at the rotor left surface and axial displacement coupling is set up at the rotor right surface.

3. Centrifugal loads of rotor and blade are introduced.

4. Temperature loads are introduced.

\section{Monitoring points}

Analysis on temperature field and stress field are conducted at different start-stop condition. According to the results, some stress concentration areas are selected as main monitoring point for subsequent analysis. The detailed monitoring points are defined as Fig. 2.

1. A1 is the rotor outer side transitional round corner before the governing stage; A2 is the corresponding axial node.

2. B1 is the rotor outer side transitional round corner after the governing stage; B2 is the corresponding axial node.

3. C1 is the circular section at the front shaft seal; C2 is the corresponding axial node.

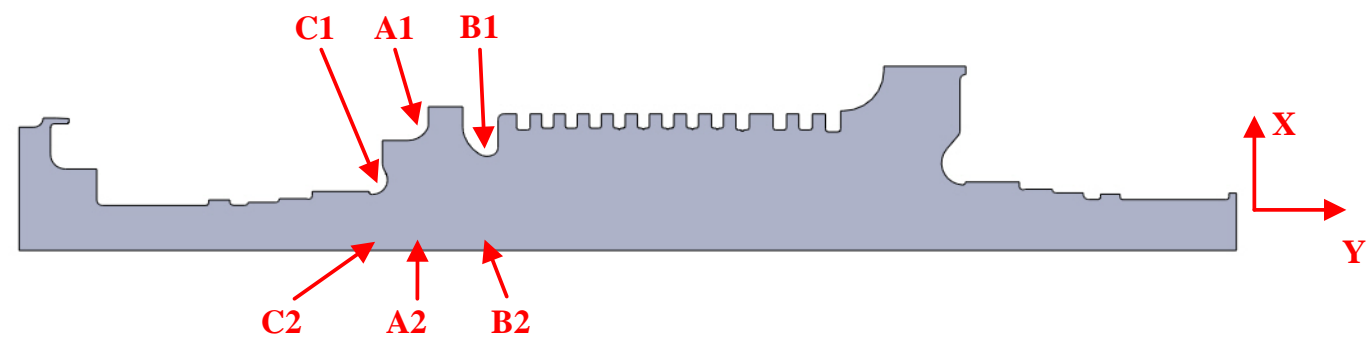

Fig. 2 Position of monitoring points

\section{Results and Discussion}

The steady-state temperature field of rotor in design condition is shown in Fig. 3. According to the temeprature distribution, the overall temperature distribution is that the temperature is gradually decreasing from high pressure admission side to two side. Radial temperature is almost constant. The maximum temperature area is the rotor surface at the governing stage. 


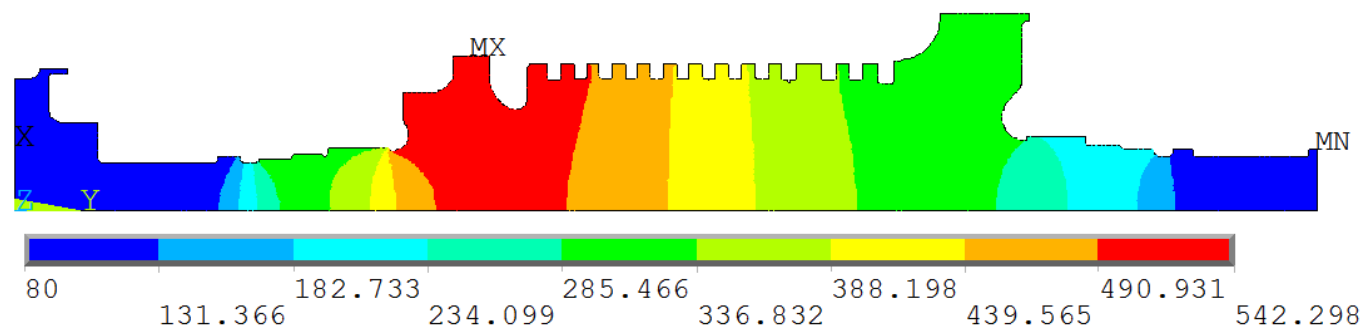

Fig. 3 Temperature distribution on design condition $/{ }^{\circ} \mathrm{C}$

The downtime process of power plant undergoes around 330 minutes. The temperature curves with time on rotor monitoring points are shown in Fig. 4 (a), (b) and (c). At the start of downtime, the rotor surface temperature remains unchanged because the main steam temperature is still. After 32 minutes, the mainstream temperature and reheated steam temperature are decreasing dramatically which lead to fast decrease of rotor surface temperature while the rotor heat exchange in radial direction is small. Overall, some lag is existed between the core temperature and surface temperature. After 172 minutes, the mainstream temperature and pressure are kept constant. In this case, the temperatures on monitoring points are relatively unchanged. At this period, the core temperature of rotor is decreasing with time. Due to the effect of heat conduction, the temperature of rotor surface and core are more and more approaching.

Fig. 4(d) is the temperature curve with time on rotor monitoring points. The temperature differences between surface and core on different monitoring points are similar. During the start of downtime, the core temperature of monitoring point A and B is lower than the surface temperature. Following this, the rotor surface temperature is decreasing rapidly while core temperature is changing slowly which is higher than the surface temperature. With the effect of heat conduction, the temperature difference is decreasing gradually.

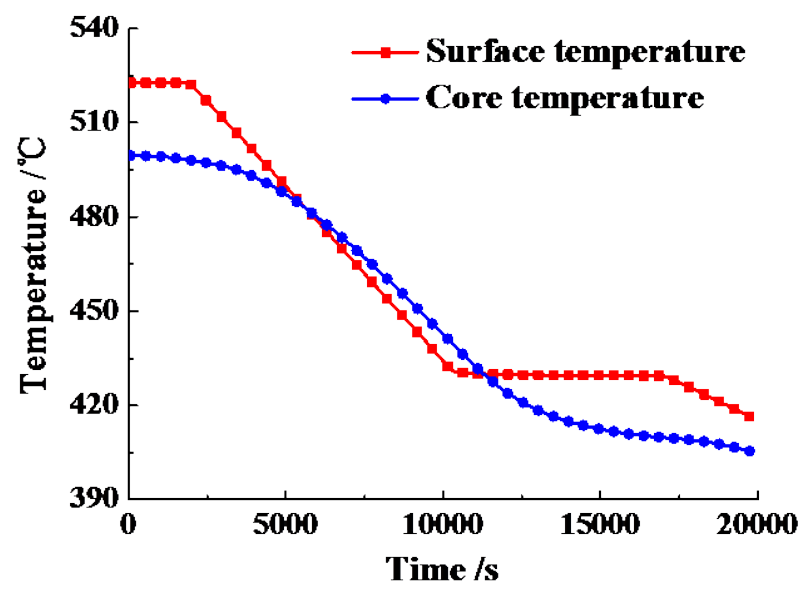

(a) Monitoring point $\mathrm{A}$



(c) Monitoring point $\mathrm{C}$

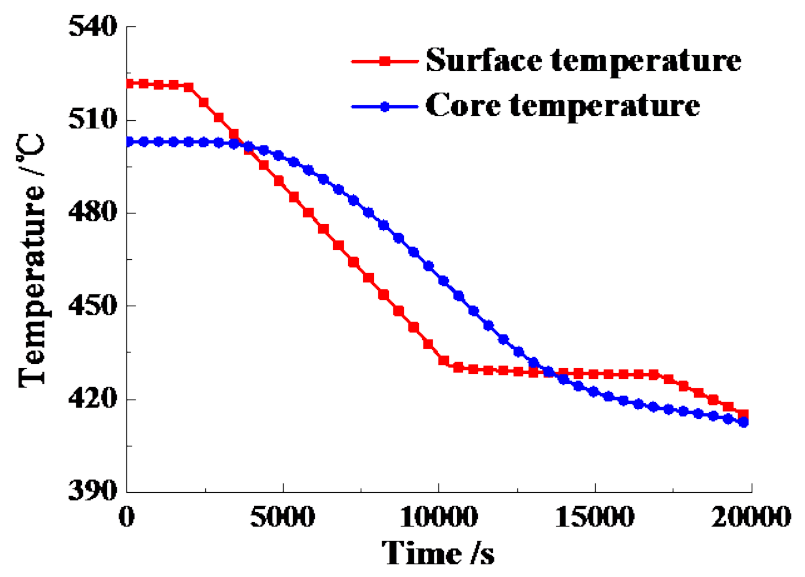

(b) Monitoring point B

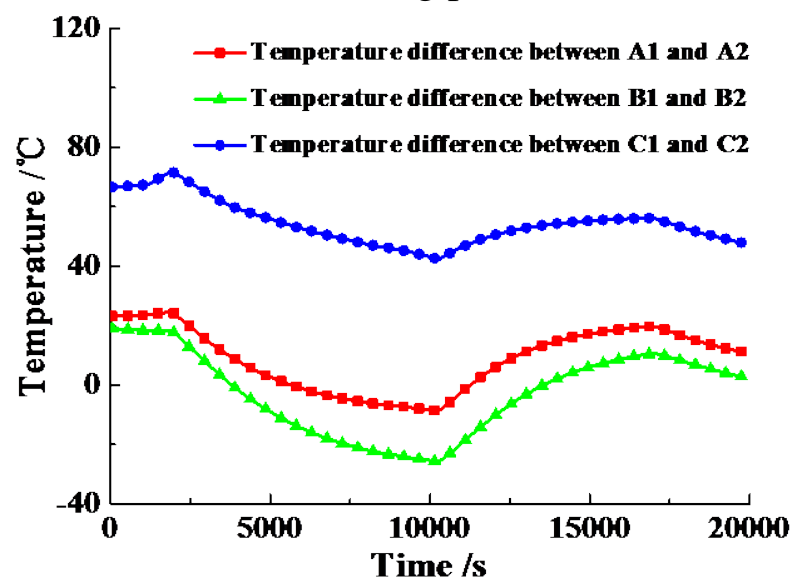

(d) Temperature difference

Fig.4 Temperature and temperature difference on different monitoring points 
Fig. 5 is the equivalent stress on monitoring points during the downtime at sliding parameters. During the start of downtime, the rotor surface temperature is rapidly decreasing under the change of mainstream temperature. Tensile stress is existed at the rotor outer surface. The stress level of rotor surface is increasing when it overlays with the rotor centrifugal force. After this, the rotor surface temperature and core temperature are approaching and decreasing under the effect of heat conduction. The maximum stress during the downtime process exists at monitoring point C1 which is $282 \mathrm{Mpa}$. The shape changes suddenly in this area which leads to large stress by the centrifugal force. Besides this, the temperature changes rapidly which make this area the dangerous point during the downtime process.



(a) Outer surface

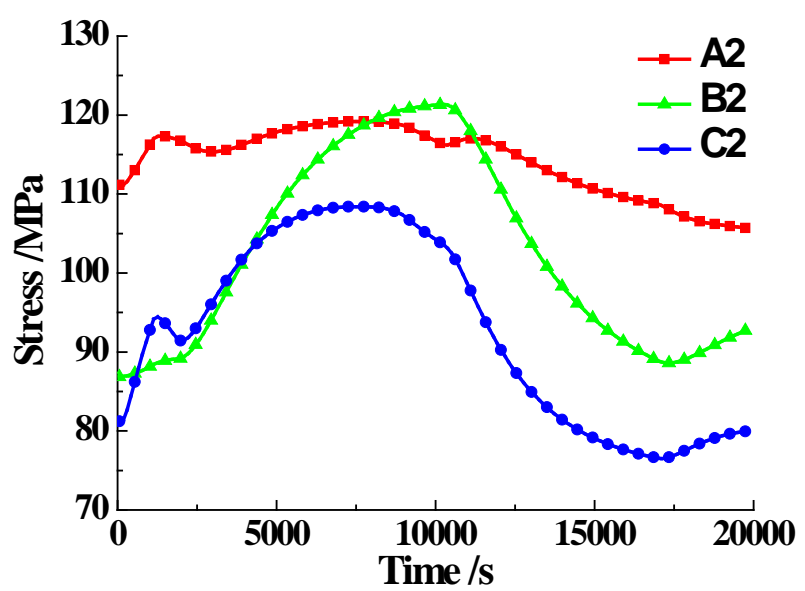

(b) Center

Fig. 5 Equivilent stress on different monitoring points during the downtime process

\section{Conclusions}

Based on a high pressure rotor, the transient temperature field and stress field during the downtime process are calculated in detial using software ANSYS. The results indicate that, shape mutation exists in the circular section near the front shaft seal which lead to large stress level under the effect of centrifugal force. Besides this, the temperature changes dramatically which causes the area the most dangerous where cracks easily occurs.

\section{Reference}

[1] Zhang Baoheng. Large-capacity thermal power unit management and peak shaving operation[M]. Beijing: Water Resources and Electric Power Press, 1988: 1-3 (In Chinese).

[2] Wang Kun. Large steam turbine rotor life problem study[D]. Wuhan: Huazhong University of Science and Technology, 2004 (In Chinese).

[3] Bai Yun. 600MW Steam turbine rotor low cycle fatigue life calculation and research[D]. Changsha: Changsha University of Science and Technology, 2009 (In Chinese).

[4] Zhen Lipeng. Combined-cycle steam turbine rotor thermal stress calculation and start the optimization research based on APDL[D]. Hangzhou: Zhejiang University, 2011 (In Chinese).

[5] Wu Wenrui. Ultra supercritical steam turbine rotor high temperature strength[D]. Shanghai: Shanghai Jiaotong University, 2009 (In Chinese).

[6] Ding Youyu. Steam turbine strength calculation manual[M]. Beijing: China Electric Power Press, 2010: 396-405 (In Chinese). 